\title{
FREQUENCY OF $a$-POINTS FOR THE FIFTH AND THE THIRD PAINLEVÉ TRANSCENDENTS IN A SECTOR
}

\author{
SHUN SHIMOMURA
}

(Received December 18, 2012)

\begin{abstract}
For the fifth Painlevé transcendents in a sector, under the condition that the values taken along some curve tending to infinity are bounded away from 1 and another specified complex number, we present new upper estimates for the number of $a$-points including poles and for the growth order. As far as we are concerned with the known asymptotic solutions of the fifth Painlevé equation, this condition is easily checked, and our results are applicable to almost all of them. About concrete examples we discuss the frequency of $a$-points, the equi-distribution property and the growth order. Our method works on the third Painlevé transcendents as well, yielding an analogous result.
\end{abstract}

1. Introduction. The fifth Painlevé equation may be normalised as follows:

$$
\begin{aligned}
w^{\prime \prime}= & \left(\frac{1}{2 w}+\frac{1}{w-1}\right)\left(w^{\prime}\right)^{2}-\frac{w^{\prime}}{z} \\
& +\frac{(w-1)^{2}}{z^{2}}\left(\alpha w+\frac{\beta}{w}\right)+\frac{\gamma w}{z}+\frac{w(w+1)}{2(w-1)}
\end{aligned}
$$

or

$\left(\mathrm{V}_{0}\right)$

$$
w^{\prime \prime}=\left(\frac{1}{2 w}+\frac{1}{w-1}\right)\left(w^{\prime}\right)^{2}-\frac{w^{\prime}}{z}+\frac{(w-1)^{2}}{z^{2}}\left(\alpha w+\frac{\beta}{w}\right)-\frac{w}{8 z}
$$

(' $=d / d z$ ) with $\alpha, \beta, \gamma \in \boldsymbol{C}$, except for the cases solvable by quadrature. Every solution of (V) or $\left(\mathrm{V}_{0}\right)$ is meromorphic on $\mathcal{R}(\boldsymbol{C} \backslash\{0\})$, where $\mathcal{R}(\boldsymbol{C} \backslash\{0\})$ denotes the universal covering of $C \backslash\{0\}$. For solutions of the fifth and the third Painlevé equations, value distribution properties were studied by Sasaki [6], [7] (see also [11] for their modified versions). From them it follows that, for every $a \in C \cup\{\infty\}$, the number of $a$-points in a sector with radius $r$ is at most $O\left(r^{\Lambda}\right)$, where $\Lambda$ is some large positive number independent of $a$; and, for admissible or truncated solutions, we established the equi-distribution property of $a$-points in a sector [12], [13].

In this paper, for solutions of $(\mathrm{V})$ or $\left(\mathrm{V}_{0}\right)$, under the condition that the values taken along some curve tending to $z=\infty$ are bounded away from 1 and another specified complex number, we present new upper estimates for the number of $a$-points and for the growth order in a sector containing the curve. It is shown that, for such solutions, the number of $a$-points is $O\left(r^{2}\right)$ or $O(r)$, which has been conjectured and is expected to be the best possible for a

2010 Mathematics Subject Classification. Primary 34M55; Secondary 34M05, 30D35.

Key words and phrases. Painlevé equations, asymptotic solution, value distribution, growth order. 
general solution [3]. For $(\mathrm{V})$ and $\left(\mathrm{V}_{0}\right)$ several asymptotic solutions are known near $z=\infty$. As far as we are concerned with these solutions, the condition mentioned above is easily checked, and our results are applicable to almost all of them. About concrete examples we discuss the frequency of $a$-points, the equi-distribution property and the growth order.

To prove our theorems for $(\mathrm{V})$ or $\left(\mathrm{V}_{0}\right)$, we first evaluate the frequency of 1-points in Section 3. As shown in Section 4, this enables us to derive an estimate for the growth order, which brings about the result on $a$-points for $a \neq 1$. Our method works on the third Painlevé equation as well, yielding an analogous theorem described in the final section. Throughout this paper, for functions $\psi$ and $\chi$, we write $\psi \ll \chi$ if $\psi=O(\chi)$, and $\psi \asymp \chi$ if $\psi=O(\chi)$ and $\chi=O(\psi)$ simultaneously hold.

2. Main results on $(\mathbf{V})$ or $\left(\mathbf{V}_{0}\right)$. Let $\mu$ and $\theta_{0}$ be given numbers satisfying $\mu \in \boldsymbol{C} \backslash\{1\}$ and $\theta_{0} \in \boldsymbol{R}$. For any solution $w(z)$ of $(\mathrm{V})$ or $\left(\mathrm{V}_{0}\right)$, we say that $w(z)$ satisfies the condition $\boldsymbol{A}\left(\mu, \theta_{0}\right)$ if there exists a curve $\Gamma_{0}\left(\theta_{0}\right): z=r e^{i \theta(r)}, r \geq 1$ such that $\theta(r) \rightarrow \theta_{0}$ as $r \rightarrow+\infty$ and that $1 /(w(z)-\mu)$ and $1 /(w(z)-1)$ are bounded along $\Gamma_{0}\left(\theta_{0}\right)$.

2.1. Frequency of $a$-points. For $a \in C \cup\{\infty\}$ and for $\lambda>0$ let $v_{\lambda}^{\theta_{0}}(r, a, w)$ denote the number of $a$-points of $w(z)$ in the sector

$$
\Sigma\left(\theta_{0}, \lambda, r\right): \quad\left|\arg \left(z-e^{i \theta_{0}} / 2\right)-\theta_{0}\right|<\lambda \pi / 2, \quad 1<\left|z-e^{i \theta_{0}} / 2\right| \leq r
$$

contained in $\mathcal{R}(\boldsymbol{C} \backslash\{0\})$, each counted according to its multiplicity.

THEOREM 2.1. Let $w(z)$ be a solution of $(\mathrm{V})$ (respectively, $\left.\left(\mathrm{V}_{0}\right)\right)$ satisfying $\boldsymbol{A}\left(\mu, \theta_{0}\right)$ for some $\left(\mu, \theta_{0}\right) \in(\boldsymbol{C} \backslash\{0, \pm 1\}) \times \boldsymbol{R}$ (respectively, $\left.\in(\boldsymbol{C} \backslash\{0,1\}) \times \boldsymbol{R}\right)$. Then, for each $a \in \boldsymbol{C} \cup\{\infty\}$ and for each $\lambda>0$,

$$
\left.v_{\lambda}^{\theta_{0}}(r, a, w) \ll r^{2} \quad \text { (respectively, } v_{\lambda}^{\theta_{0}}(r, a, w) \ll r\right)
$$

as $r \rightarrow+\infty$, where the implied constant depends on $\lambda$.

2.2. Growth order. For every $r>1$ consider the sector-like set

$$
\Omega\left(\theta_{0}, \lambda, r\right):=\left\{z=e^{i \theta_{0}}\left(e^{-\pi i / 2} \zeta\right)^{\lambda}+e^{i \theta_{0}} / 2 ; \zeta \in D_{0}\left(r^{1 / \lambda}\right)\right\} \subset \mathcal{R}(\boldsymbol{C} \backslash\{0\})
$$

with $D_{0}(r):=\{\zeta ;|\zeta-i r / 2| \leq r / 2\} \cap\{\zeta ;|\zeta|>1\}$. Roughly speaking, this set is a substitute for the sector $\Sigma\left(\theta_{0}, \lambda, r\right)$. Indeed, for every $\lambda^{\prime}$ satisfying $0<\lambda^{\prime}<\lambda$,

$$
\Sigma\left(\theta_{0}, \lambda^{\prime}, r \cos ^{\lambda}\left(\lambda^{\prime} \pi /(2 \lambda)\right)\right) \subset \Omega\left(\theta_{0}, \lambda, r\right) \subset \Sigma\left(\theta_{0}, \lambda, r\right),
$$

where the left inclusion is verified by using

$$
\left\{\zeta ;|\arg \zeta-\pi / 2|<\lambda^{\prime} \pi /(2 \lambda), 1<|\zeta|<r^{1 / \lambda} \cos \left(\lambda^{\prime} \pi /(2 \lambda)\right)\right\} \subset D_{0}\left(r^{1 / \lambda}\right) .
$$

Let $f(z)$ be a function meromorphic on $\mathcal{R}(\boldsymbol{C} \backslash\{0\})$. Denote by $n_{\lambda}^{\theta_{0}}(r, f)$ the number of poles of $f(z)$ in $\Omega\left(\theta_{0}, \lambda, r\right)$, each counted according to its multiplicity. For $r>1$ set

$$
N_{\lambda}^{\theta_{0}}(r, f):=\frac{1}{\lambda} \int_{1}^{r} \frac{n_{\lambda}^{\theta_{0}}(t, f)}{t^{1+1 / \lambda}} d t
$$


and

$$
\begin{aligned}
m_{\lambda}^{\theta_{0}}(r, f) & :=\frac{1}{2 \pi} \int_{\arcsin \left(r^{-1 / \lambda}\right)}^{\pi-\arcsin \left(r^{-1 / \lambda}\right)} \frac{\log ^{+}\left|f_{\lambda}^{\theta_{0}}\left(r^{1 / \lambda} e^{i \phi} \sin \phi\right)\right|}{r^{1 / \lambda} \sin ^{2} \phi} d \phi \\
f_{\lambda}^{\theta_{0}}(\zeta) & :=f\left(e^{i \theta_{0}}\left(e^{-\pi i / 2} \zeta\right)^{\lambda}+e^{i \theta_{0}} / 2\right)
\end{aligned}
$$

where $\log ^{+} x:=\max \{\log x, 0\}$ for $x>0$. The characteristic function defined by

$$
T_{\lambda}^{\theta_{0}}(r, f):=m_{\lambda}^{\theta_{0}}(r, f)+N_{\lambda}^{\theta_{0}}(r, f)
$$

indicates the growth of $f(z)$ in $\Omega\left(\theta_{0}, \lambda, r\right)$ or $\Sigma\left(\theta_{0}, \lambda, r\right)$. Furthermore, set

$$
\varrho_{\lambda}^{\theta_{0}}(f):=\limsup _{r \rightarrow \infty} \frac{\log ^{+} T_{\lambda}^{\theta_{0}}(r, f)}{\log r}, \quad \sigma_{\lambda}^{\theta_{0}}(a, f):=\limsup _{r \rightarrow \infty} \frac{\log ^{+} N_{\lambda}^{\theta_{0}}(r, a, f)}{\log r},
$$

where $N_{\lambda}^{\theta_{0}}(r, \infty, f):=N_{\lambda}^{\theta_{0}}(r, f)$ and $N_{\lambda}^{\theta_{0}}(r, a, f):=N_{\lambda}^{\theta_{0}}(r, 1 /(f-a))$ for $a \in \boldsymbol{C}$. (More explanations of these quantities are found in [12] and [13]; and, for the value distribution theory in a half plane or a sector, readers are referred to [4], [5], [15], [16].)

THEOREM 2.2. Suppose that a solution $w(z)$ of $(\mathrm{V})$ (respectively, $\left(\mathrm{V}_{0}\right)$ ) satisfies the same condition as in Theorem 2.1. Then, for each $\lambda>0$,

$$
\left.T_{\lambda}^{\theta_{0}}(r, w) \ll r^{2-1 / \lambda}+\log r \quad \text { (respectively, } \quad T_{\lambda}^{\theta_{0}}(r, w) \ll r^{1-1 / \lambda}+\log r\right)
$$

as $r \rightarrow+\infty$, where the implied constant depends on $\lambda$; and, for every $a \in C \cup\{\infty\}$, $\sigma_{\lambda}^{\theta_{0}}(a, w) \leq \max \{2-1 / \lambda, 0\}$ (respectively, $\sigma_{\lambda}^{\theta_{0}}(a, w) \leq \max \{1-1 / \lambda, 0\}$ ).

This estimate means that, for the significance of $T_{\lambda}^{\theta_{0}}(r, w)$, the opening angle of the sector must satisfy at least $\lambda>1 / 2$ (respectively, $\lambda>1$ ).

2.3. Asymptotic solutions. Each of the known asymptotic solutions of (V) (respectively, $\left(\mathrm{V}_{0}\right)$ ), except for ones with special constants of integration [2, Theorem 4.1 and Remark $4.2]$, tends to a value $w_{\infty} \in\{-1,0, \infty\}$ (respectively, $w_{\infty} \in\{0, \infty\}$ ) or oscillates around $w_{\infty}$ as $z \rightarrow \infty$ along a certain ray or curve (see [1], [2], [8], [9], [13], [14], [17]). For these solutions we have $\nu_{\lambda}^{\theta_{0}}(r, a, w) \ll r^{2}$ (respectively, $\ll r$ ) and $T_{\lambda}^{\theta_{0}}(r, w) \ll r^{2-1 / \lambda}+\log r$ (respectively, $\ll r^{1-1 / \lambda}+\log r$ ) with some $\theta_{0} \in \boldsymbol{R}$. Let us observe individual examples.

2.3.1. Solutions of $(\mathbf{V})$. A two-parameter solution of $(\mathrm{V})$ equivalent to one given by $[2$, Theorem 3.1] is written in the form

$$
w_{1}(z)=-1+4 \sqrt{\frac{2 i}{z}} e^{-\pi i / 4} \sqrt{\beta_{0}} \sin \left(\frac{z}{2 i}+i \beta_{0} \log \left(\frac{z}{i}\right)-i \log \left(\frac{\sqrt{\beta_{0}}}{\hat{v}}\right)\right)+O\left(z^{-1+2\left|\operatorname{Re} \beta_{0}\right|}\right)
$$

as $z / i \rightarrow+\infty$, where $\beta_{0}, \hat{v} \in C \backslash\{0\},\left|\operatorname{Re} \beta_{0}\right|<1 / 4$ (note that, in the equation of [2], the coefficient of $w(w+1) /(w-1)$ is $-1 / 2)$. This satisfies $\boldsymbol{A}(-2, \pi / 2)$. There also exist the oscillatory solutions [9, Theorems I and III]:

$$
w_{2}^{-}(z)=\rho\left(1+O\left(z^{-1}\right)\right) z^{-1} \cos ^{2}\left(z / 2-(\gamma / 2-\rho / 2) \log z+\Theta_{0}+O\left(z^{-1}\right)\right)
$$


as $z \rightarrow+\infty$ if $\alpha, \gamma \in \boldsymbol{R}, \beta=0$; and

$$
w_{2}^{+}(z)=\frac{\rho\left(1+O\left(z^{-1}\right)\right) z}{\cos ^{2}\left(z / 2+\left(\gamma / 2+\rho^{-1} / 2\right) \log z+\Theta_{0}+O\left(z^{-1}\right)\right)}
$$

as $z \rightarrow+\infty$ if $\alpha=\beta=0, \gamma \in \boldsymbol{R}$. Here $\rho>0$ and $0 \leq \Theta_{0}<2 \pi$ are constants of integration. These solutions satisfy $\boldsymbol{A}(-2,0)$.

A family of solutions expanded into

$$
\begin{aligned}
w_{3}(z)= & Y(z)+\sum_{j \geq k+1 \geq 1} z^{-1} p_{j k}(z) Y(z)^{j-k}(Y(z) Z(z))^{k} \\
& +\sum_{k \geq j \geq 0} z^{-1} p_{j k}(z)(Y(z) Z(z))^{j}\left(z^{-1} Z(z)\right)^{k-j}
\end{aligned}
$$

with

$$
Y(z):=c_{1} z^{-\omega-4 c_{1} c_{2}} e^{-i z}, \quad Z(z):=c_{2} z^{\omega+4 c_{1} c_{2}} e^{i z}, \quad \omega:=1-i(\gamma-2 \sqrt{2 \beta})
$$

follows from the results of [8], [14], [17], where $c_{1}, c_{2} \in \boldsymbol{C}$ are constants of integration, and each $p_{j k}(z)$ admits an asymptotic expansion in powers of $z^{-1}$ in the sector $|\arg z|<\pi$. The right-hand side is convergent if $|Y(z)|,\left|z^{-1} Z(z)\right|$ and $|Y(z) Z(z)|=\left|c_{1} c_{2}\right|$ are sufficiently small, and is bounded as $z \rightarrow \infty$ along a curve asymptotic to the positive real axis. This solution satisfies $\boldsymbol{A}(-2,0)$.

Equation (V) has a truncated solution of the form

$$
w_{4}(z)=-1+O\left(z^{-1}\right)+c e^{-z / 2} z^{-1 / 2}\left(1+O\left(z^{-1}\right)\right)
$$

with $c \in C$ as $z \rightarrow \infty$ through $|\arg z|<\pi / 2$ [13, Theorem 2.1]. If $\left|\theta_{0}\right|<\pi / 2$, then $w_{4}(z)$ satisfies $\boldsymbol{A}\left(-2, \theta_{0}\right)$. There also exist truncated solutions such that $w(z) \rightarrow 0$ and that $w(z) \rightarrow \infty$, respectively; for instance, if $\alpha \neq 0$, then (V) admits the solution

$$
w_{5}(z)=\frac{z}{\sqrt{-2 \alpha}}\left(1+O\left(z^{-1}\right)\right)+c e^{i z} z^{(\gamma+2 \sqrt{-2 \alpha}) i+1}\left(1+O\left(z^{-1}\right)\right)
$$

as $z \rightarrow \infty$ through $|\arg z-\pi / 2|<\pi / 2$ [13, Theorem 2.4], which satisfies $\boldsymbol{A}\left(-2, \theta_{0}\right)$ if $\left|\theta_{0}-\pi / 2\right|<\pi / 2$.

Applying Theorems 2.1 and 2.2, we have, for each $a \in C \cup\{\infty\}$, the following:

PROPOSITION 2.3. (1) $v_{\lambda}^{\pi / 2}\left(r, a, w_{1}\right) \ll r^{2}$ and $T_{\lambda}^{\pi / 2}\left(r, w_{1}\right) \ll r^{2-1 / \lambda}+\log r$.

(2) $v_{\lambda}^{0}\left(r, a, w_{2}^{ \pm}\right) \ll r^{2}$ and $T_{\lambda}^{0}\left(r, w_{2}^{ \pm}\right) \ll r^{2-1 / \lambda}+\log r$.

(3) $v_{\lambda}^{0}\left(r, a, w_{3}\right) \ll r^{2}$ and $T_{\lambda}^{0}\left(r, w_{3}\right) \ll r^{2-1 / \lambda}+\log r$.

(4) If $\left|\theta_{0}\right|<\pi / 2$, then $v_{\lambda}^{\theta_{0}}\left(r, a, w_{4}\right) \ll r^{2}$ and $T_{\lambda}^{\theta_{0}}\left(r, w_{4}\right) \ll r^{2-1 / \lambda}+\log r$.

(5) If $\left|\theta_{0}-\pi / 2\right|<\pi / 2$, then $v_{\lambda}^{\theta_{0}}\left(r, a, w_{5}\right) \ll r^{2}$ and $T_{\lambda}^{\theta_{0}}\left(r, w_{5}\right) \ll r^{2-1 / \lambda}+\log r$.

Furthermore, combining (2) and (4) with [12, Example 2.2] and [13, Theorem 3.2, Remark 3.2 and (6.3)], respectively, we have the following equi-distribution estimates:

Proposition 2.4. If $\lambda>1$, then $r^{1-1 / \lambda} \ll T_{\lambda}^{0}\left(r, w_{2}^{ \pm}\right) \ll r^{2-1 / \lambda}$ and $1-1 / \lambda \leq$ $\sigma_{\lambda}^{0}\left(a, w_{2}^{ \pm}\right)=\varrho_{\lambda}^{0}\left(w_{2}^{ \pm}\right) \leq 2-1 / \lambda$ for every $a \in \boldsymbol{C} \cup\{\infty\}$. 
Proposition 2.5. Suppose that $0<\theta_{0}<\pi / 2$, and that $\lambda>1$. Write $\lambda_{0}=1+$ $2 \theta_{0} / \pi$, and set $l(\lambda)=\lambda$ if $1<\lambda<\lambda_{0}$ and $l(\lambda)=\lambda_{0}-\varepsilon$ if $\lambda \geq \lambda_{0}$, where $\varepsilon$ is any small positive number. Then, if $c \neq 0$, we have $r^{1-1 / l(\lambda)} \ll T_{\lambda}^{\theta_{0}}\left(r, w_{4}\right) \ll r^{2-1 / \lambda}$ and $1-1 / l(\lambda) \leq \sigma_{\lambda}^{\theta_{0}}\left(a, w_{4}\right)=\varrho_{\lambda}^{\theta_{0}}\left(w_{4}\right) \leq 2-1 / \lambda$ for every $a \in \boldsymbol{C} \cup\{\infty\}$.

2.3.2. Solutions of $\left(\mathrm{V}_{0}\right)$. Equation $\left(\mathrm{V}_{0}\right)$ has the oscillatory solutions [9, Theorems II and IV]:

$$
w_{6}^{-}(z)=\rho\left(1+O\left(z^{-1 / 2}\right)\right) z^{-1 / 2} \cos ^{2}\left(z^{1 / 2} / 2+(\rho / 16) \log z+\Theta_{0}+O\left(z^{-1 / 2}\right)\right)
$$

as $z \rightarrow+\infty$ if $\alpha \in \boldsymbol{R}, \beta=0$; and

$$
w_{6}^{+}(z)=\frac{\rho\left(1+O\left(z^{-1 / 2}\right)\right)(-z)^{1 / 2}}{\cos ^{2}\left((-z)^{1 / 2} / 2+\left(\rho^{-1} / 16\right) \log (-z)+\Theta_{0}+O\left(z^{-1 / 2}\right)\right)}
$$

as $z \rightarrow-\infty$ if $\alpha=\beta=0$. Here $\rho>0$ and $0 \leq \Theta_{0}<2 \pi$. These solutions satisfy $\boldsymbol{A}(-1,0)$ and $\boldsymbol{A}(-1, \pi)$, respectively.

There exists a truncated solution of $\left(\mathrm{V}_{0}\right)$ expressed as

$$
w_{7}(z)=2 \sqrt{2 \beta} z^{-1 / 2}\left(1+O\left(z^{-1 / 2}\right)\right)+c e^{i z^{1 / 2}} z^{\sqrt{\beta / 2} i-1 / 2}\left(1+O\left(z^{-1 / 2}\right)\right)
$$

with $c \in \boldsymbol{C}$ as $z \rightarrow \infty$ through $|\arg z-\pi|<\pi$ [13, Theorem 2.3], which satisfies $\boldsymbol{A}\left(-1, \theta_{0}\right)$ if $\left|\theta_{0}-\pi\right|<\pi$. For each $a \in C \cup\{\infty\}$ we have

PROPOSITION 2.6. (1) $v_{\lambda}^{0}\left(r, a, w_{6}^{-}\right) \ll r$ and $T_{\lambda}^{0}\left(r, w_{6}^{-}\right) \ll r^{1-1 / \lambda}+\log r$.

(2) $v_{\lambda}^{\pi}\left(r, a, w_{6}^{+}\right) \ll r$ and $T_{\lambda}^{\pi}\left(r, w_{6}^{+}\right) \ll r^{1-1 / \lambda}+\log r$.

(3) If $\left|\theta_{0}-\pi\right|<\pi$, then $v_{\lambda}^{\theta_{0}}\left(r, a, w_{7}\right) \ll r$ and $T_{\lambda}^{\theta_{0}}\left(r, w_{7}\right) \ll r^{1-1 / \lambda}+\log r$.

By (1) above and [12, Example 2.3] we have

PROPOSITION 2.7. If $\lambda>2$, then $r^{1 / 2-1 / \lambda} \ll T_{\lambda}^{0}\left(r, w_{6}^{-}\right) \ll r^{1-1 / \lambda}$ and $1 / 2-1 / \lambda \leq$ $\sigma_{\lambda}^{0}\left(a, w_{6}^{-}\right)=\varrho_{\lambda}^{0}\left(w_{6}^{-}\right) \leq 1-1 / \lambda$ for every $a \in C \cup\{\infty\}$.

\section{Proof of Theorem 2.1 for $a=1$.}

3.1. Lemmas. Consider a differential equation of the form

$$
\ddot{y}=g_{2}(y) \dot{y}^{2}+g_{1}(x) \dot{y}+1+g_{0}(x, y)
$$

$(\cdot=d / d x)$ under the supposition that $g_{2}(y), g_{1}(x)$ and $g_{0}(x, y)$ are holomorphic functions satisfying

$$
\left|g_{2}(y)\right|<K, \quad\left|g_{1}(x)\right|+\left|g_{0}(x, y)\right|<1 / 100
$$

in the polydisk $|x|<1,|y|<R_{0}$, where $0<R_{0}<1$ and $K>1$. Let $y(x)$ be an arbitrary solution of (3.1) around $x=0$. Then we have

LEMMA 3.1. Let $\eta$ be a given number such that $0<\eta \leq \min \left\{R_{0}^{1 / 2} / 3, K^{-1 / 2} / 30\right\}$. If $|y(0)| \leq \eta^{2} / 6$, then

(1) $|y(x)| \leq 8 \eta^{2}<R_{0}$ in the disk $|x|<\rho_{0}$,

(2) $|y(x)| \geq \eta^{2} / 5$ on the circle $|x|=(5 / 6) \rho_{0}$.

Here $\rho_{0}:=3 \eta$ if $|\dot{y}(0)| \leq \eta$, and $\rho_{0}:=1.2 \eta^{2} /|\dot{y}(0)|$ if $|\dot{y}(0)|>\eta$. 
ProOF. If $|x|<1$ and if $|y(x)|<R_{0}$, then

$$
\dot{y}(x)=\dot{y}(0)+x+\dot{V}(x)
$$

and

$$
y(x)=y(0)+\dot{y}(0) x+x^{2} / 2+V(x),
$$

where

$$
V(x)=\int_{0}^{x} \int_{0}^{\tilde{\xi}}\left(g_{2}(y(\xi)) \dot{y}(\xi)^{2}+g_{1}(\xi) \dot{y}(\xi)+g_{0}(\xi, y(\xi))\right) d \xi d \tilde{\xi}
$$

(i) Case $|\dot{y}(0)| \leq \eta$. We put

$$
\xi_{0}:=\sup \left\{\xi<1 ;|y(x)| \leq 8 \eta^{2},|\dot{y}(x)| \leq 5 \eta \text { for }|x|<\xi\right\},
$$

which satisfies $\xi_{0}>0$ because of $|y(0)| \leq \eta^{2} / 6$ and $|\dot{y}(0)| \leq \eta$. Suppose that $\xi_{0}<3 \eta$. Then $|y(x)|<R_{0}$ for $|x|<\xi_{0} \leq 1$. By (3.2) and (3.5), for $|x|<\xi_{0}$,

$$
|\dot{V}(x)| \leq\left(K(5 \eta)^{2}+5 \eta / 100+1 / 100\right)|x| \leq|x| / 15, \quad|V(x)| \leq|x|^{2} / 30 .
$$

From (3.6), (3.3) and (3.4) we have

$$
\begin{aligned}
& |\dot{y}(x)| \leq(1+3+1 / 5) \eta=4.2 \eta, \\
& |y(x)| \leq\left(1 / 6+3+3^{2} / 2+3^{2} / 30\right) \eta^{2}<7.97 \eta^{2}
\end{aligned}
$$

for $|x|<\xi_{0}$, which contradicts the definition of $\xi_{0}$. Hence $\xi_{0} \geq 3 \eta$; and (3.6) is valid for $|x|<3 \eta$. On the circle $|x|=2.5 \eta$, from (3.4) we derive

$$
\begin{aligned}
|y(x)| & \geq|x|^{2} / 2-|y(0)|-|\dot{y}(0) x|-|V(x)| \\
& \geq\left(2.5^{2} / 2-1 / 6-2.5-2.5^{2} / 30\right) \eta^{2}>\eta^{2} / 5 .
\end{aligned}
$$

Consequently assertions (1) and (2) are valid for $\rho_{0}=3 \eta$.

(ii) Case $|\dot{y}(0)|=\kappa \eta$ with $\kappa>1$. Put

$$
\xi_{1}:=\sup \left\{\xi<1 ;|y(x)| \leq 5 \eta^{2},|\dot{y}(x)| \leq 4 \kappa \eta \text { for }|x|<\xi\right\},
$$

and suppose that $\xi_{1}<2 \eta / \kappa$. Then we have

(3.7) $|\dot{V}(x)| \leq\left(K(4 \kappa \eta)^{2}+4 \kappa \eta / 100+1 / 100\right)|x| \leq \kappa^{2}|x| / 15, \quad|V(x)| \leq \kappa^{2}|x|^{2} / 30$ for $|x|<\xi_{1}$. By (3.7), (3.3) and (3.4),

$$
\begin{aligned}
& |\dot{y}(x)| \leq \kappa \eta+2 \eta / \kappa+\left(\kappa^{2} / 15\right)(2 \eta / \kappa)<3.5 \kappa \eta, \\
& |y(x)| \leq \eta^{2} / 6+\kappa \eta \cdot 2 \eta / \kappa+(2 \eta / \kappa)^{2} / 2+\left(\kappa^{2} / 30\right)(2 \eta / \kappa)^{2}<4.5 \eta^{2}
\end{aligned}
$$

for $|x|<\xi_{1}$, which contradicts the definition of $\xi_{1}$. Hence $\xi_{1} \geq 2 \eta / \kappa$; and (3.7) is valid for $|x|<2 \eta / \kappa$. On the circle $|x|=\eta / \kappa$,

$$
\begin{aligned}
|y(x)| & \geq|\dot{y}(0) x|-|y(0)|-|x|^{2} / 2-|V(x)| \\
& \geq(1-1 / 6-1 / 2-1 / 30) \eta^{2}>\eta^{2} / 5 .
\end{aligned}
$$

In this way we obtain (1) and (2) with $\rho_{0}=1.2 \eta / \kappa=1.2 \eta^{2} /|\dot{y}(0)|$, which completes the proof. 
LEMMA 3.2. Let $w(z)$ be a solution of $(\mathrm{V})$ (respectively, $\left.\left(\mathrm{V}_{0}\right)\right)$ with $\boldsymbol{A}\left(\mu, \theta_{0}\right)$ as in Theorem 2.1 along the curve $\Gamma_{0}\left(\theta_{0}\right)$, and set $\Delta_{0}:=\inf \left\{|w(z)-\mu| ; z \in \Gamma_{0}\left(\theta_{0}\right)\right\}$. Then there exist positive numbers $T_{0}$ and $\Delta \leq \Delta_{0} / 3$ such that the following assertions are valid for every $s \in \mathcal{R}(\boldsymbol{C} \backslash\{0\})$ satisfying $|s| \geq T_{0}$ :

(1) if $|w(s)-\mu| \leq \Delta$, then $|w(z)-\mu| \geq 2 \Delta$ on the circle $|z-s|=\delta_{s}$;

(2) $w(z) \neq 1$ in the disk $|z-s| \leq \delta_{s}$.

Here $\delta_{s}$ is some positive number satisfying

$$
\begin{gathered}
\delta_{s}^{-1} \leq C_{0}\left(1+\left|w^{\prime}(s)\right|\right), \quad \delta_{s} \leq C_{0} \\
\left(\text { respectively, } \delta_{s}^{-1} \leq C_{0}|s|^{-1 / 2}\left(1+|s|^{-1 / 2}\left|w^{\prime}(s)\right|\right), \quad \delta_{s} \leq C_{0}|s|^{1 / 2} \leq|s|\right),
\end{gathered}
$$

$C_{0}>0$ being independent of $s$.

Proof. Suppose that $w(z)$ satisfies (V) and put $z=s+h x, w(z)=y(x)+\mu$. We may take $h$ such that $\left(h^{2} / 2\right) \mu(\mu+1) /(\mu-1)=1$, since $\mu \neq 0, \pm 1$. For $|x|<1$ and for $|y(x)|<\min \{|\mu|,|\mu-1|, 1\} / 2$, write the equation of $y(x)$ in the form (3.1). Set $K:=$ $1 /(2|\mu|)+1 /|\mu-1|+1$. Then, choose $T_{0}>1$ and $R_{0}<\min \{|\mu|,|\mu-1|, 1\} / 2$ in such a way that (3.2) is valid if $|s| \geq T_{0}$. Using Lemma 3.1 with

$$
\eta=\min \left\{R_{0}^{1 / 2} / 3, K^{-1 / 2} / 30,|\mu-1|^{1 / 2} / 4,\left(10 \Delta_{0} / 3\right)^{1 / 2}\right\}
$$

we can verify (1) and (2) with $\Delta=\eta^{2} / 10 \leq \Delta_{0} / 3$ and $\delta_{s}=(5 / 6) \rho_{0}|h|$. Indeed $w(z) \neq 1$ follows from $|w(z)-\mu| \leq 8 \eta^{2} \leq|\mu-1| / 2$. If $w(z)$ is a solution of $\left(\mathrm{V}_{0}\right)$, we put $z=$ $s+h_{*} x, w(z)=y(x)+\mu$, where $(-1 / 8) h_{*}^{2} \mu / s=1$. By an analogous argument with $\delta_{s}=(5 / 6) \rho_{0}\left|h_{*}\right| \asymp(5 / 6) \rho_{0}|s|^{1 / 2}$, we obtain the lemma, where the constant $T_{0}$ is retaken larger so that $\delta_{s} \leq|s|$ if necessary.

Recall the following fact (cf. [6, Lemma 1.4], [11, Lemma 2.4], see also [10]):

LEMMA 3.3. Let $w(z)$ be a solution of $(\mathrm{V})$ (respectively, $\left(\mathrm{V}_{0}\right)$ ), and let $\Gamma\left(z_{0}, z\right)$ be a curve on $\mathcal{R}(\boldsymbol{C} \backslash\{0\})$ joining $z_{0}$ to $z$. Then the function

$$
\begin{aligned}
\Phi(\mu, z):= & \frac{z^{2} w^{\prime}(z)^{2}}{w(z)(w(z)-1)^{2}}-2 \alpha w(z)+\frac{2 \beta}{w(z)}+\frac{2 \gamma z}{w(z)-1} \\
& +\frac{z^{2} w(z)}{(w(z)-1)^{2}}-\frac{2(1-\mu) z w^{\prime}(z)}{(w(z)-1)(w(z)-\mu)} \\
(\text { respectively, }:= & \frac{z^{2} w^{\prime}(z)^{2}}{w(z)(w(z)-1)^{2}}-2 \alpha w(z)+\frac{2 \beta}{w(z)} \\
& \left.-\frac{z / 4}{w(z)-1}-\frac{2(1-\mu) z w^{\prime}(z)}{(w(z)-1)(w(z)-\mu)}\right)
\end{aligned}
$$


is expressible in the form

$$
\begin{aligned}
& \Phi(\mu, z)=E\left(z_{0}, z\right) \Phi\left(\mu, z_{0}\right)-2(1-\mu)^{2}\left(\frac{w(z)}{(w(z)-\mu)^{2}}-\frac{E\left(z_{0}, z\right) w\left(z_{0}\right)}{\left(w\left(z_{0}\right)-\mu\right)^{2}}\right) \\
& -\int_{\Gamma\left(z_{0}, z\right)} \frac{E\left(z_{0}, z\right)}{E\left(z_{0}, t\right)}\left(\frac{2(1-\mu)^{3} w(t)(w(t)-1)(w(t)+\mu)}{(w(t)-\mu)^{4}}-\frac{\Xi(t, w(t))}{(w(t)-\mu)^{2}}\right) \frac{d t}{t},
\end{aligned}
$$

provided that $w(t) \neq \mu$ along $\Gamma\left(z_{0}, z\right)$. Here

$$
E\left(z_{0}, t\right):=\exp \left((1-\mu) \int_{\Gamma\left(z_{0}, t\right)} \frac{(w(\tau)-1)(w(\tau)+\mu)}{(w(\tau)-\mu)^{2}} \frac{d \tau}{\tau}\right)
$$

with $\Gamma\left(z_{0}, t\right) \subset \Gamma\left(z_{0}, z\right)$, and

$$
\begin{gathered}
\Xi(z, w):=4(1-\mu)(w-1)(\alpha \mu w-\beta)-2 \gamma z((1-2 \mu) w+\mu)+2 \mu z^{2} w \\
\quad(\text { respectively, }:=4(1-\mu)(w-1)(\alpha \mu w-\beta)+(z / 4)((1-2 \mu) w+\mu)) .
\end{gathered}
$$

Then we have

LEMMA 3.4. Let $w(z)$ be a solution of $(\mathrm{V})$ (respectively, $\left.\left(\mathrm{V}_{0}\right)\right)$ with $\boldsymbol{A}\left(\mu, \theta_{0}\right)$ as in Theorem 2.1 along $\Gamma_{0}\left(\theta_{0}\right)$. Then there exists a positive number $K_{0}$ such that $|\Phi(\mu, z)| \leq$ $K_{0}|z|^{2}$ (respectively, $|\Phi(\mu, z)| \leq K_{0}|z|$ ) along $\Gamma_{0}\left(\theta_{0}\right)$.

PROOF. If $w(z)$ is a solution of $(\mathrm{V})$, then

$$
\Psi(z):=\frac{z^{2} w^{\prime}(z)^{2}}{w(z)(w(z)-1)^{2}}-2 \alpha w(z)+\frac{2 \beta}{w(z)}+\frac{2 \gamma z}{w(z)-1}+\frac{z^{2} w(z)}{(w(z)-1)^{2}}
$$

satisfies

$$
\Psi^{\prime}(z)=\frac{2 \gamma}{w(z)-1}+\frac{2 z w(z)}{(w(z)-1)^{2}}
$$

(cf. [10, (4.5), (4.6)]), so that, for any $z \in \Gamma_{0}\left(\theta_{0}\right)$,

$$
\Psi(z)=\Psi_{0}+\int_{\Gamma_{0}\left(\theta_{0}\right)(z)}\left(\frac{2 \gamma}{w(t)-1}+\frac{2 t w(t)}{(w(t)-1)^{2}}\right) d t
$$

where $\Gamma_{0}\left(\theta_{0}\right)(z)$ denotes the part of $\Gamma_{0}\left(\theta_{0}\right)$ from its starting point to $z$, and $\Psi_{0}$ is some complex constant. Using $\boldsymbol{A}\left(\mu, \theta_{0}\right)$, from (3.13) we derive $\Psi(z) \ll|z|^{2}$ along $\Gamma_{0}\left(\theta_{0}\right)$. Then (3.12) gives

$$
\begin{aligned}
\frac{z w^{\prime}(z)}{w(z)-1} & =\left(2 \alpha w(z)^{2}-2 \beta-\frac{2 \gamma z w(z)}{w(z)-1}-\frac{z^{2} w(z)^{2}}{(w(z)-1)^{2}}+w(z) \Psi(z)\right)^{1 / 2} \\
& \ll|w(z)|+|z|\left(1+|w(z)|^{1 / 2}\right),
\end{aligned}
$$

which implies $z w^{\prime}(z)(w(z)-1)^{-1}(w(z)-\mu)^{-1} \ll|z|$. Hence, by (3.9),

$$
\Phi(\mu, z)=\Psi(z)-\frac{2(1-\mu) z w^{\prime}(z)}{(w(z)-1)(w(z)-\mu)} \ll|z|^{2}
$$

along $\Gamma_{0}\left(\theta_{0}\right)$. In case $w(z)$ satisfies $\left(\mathrm{V}_{0}\right)$, using

$$
\Psi(z):=\frac{z^{2} w^{\prime}(z)^{2}}{w(z)(w(z)-1)^{2}}-2 \alpha w(z)+\frac{2 \beta}{w(z)}-\frac{z / 4}{w(z)-1}
$$


and $\Psi^{\prime}(z)=-(1 / 4)(w(z)-1)^{-1}$, we obtain $\Psi(z) \ll|z|$ in a similar way.

3.2. Construction of a curve. Let $w(z)$ be a solution of $(\mathrm{V})$ (respectively, $\left.\left(\mathrm{V}_{0}\right)\right)$ with $\boldsymbol{A}\left(\mu, \theta_{0}\right)$ as in Theorem 2.1 along $\Gamma_{0}\left(\theta_{0}\right)$, and let $\lambda>0$ be a given number.

LEMMA 3.5. For every $\sigma \in \mathcal{R}(\boldsymbol{C} \backslash\{0\})$ satisfying $w(\sigma)=1,|\sigma| \geq T_{0}$ and $\mid \arg (\sigma-$ $\left.e^{i \theta_{0}} / 2\right)-\theta_{0} \mid \leq \lambda \pi / 2$, there exists a curve $\Gamma_{\sigma} \subset \mathcal{R}(\boldsymbol{C} \backslash\{0\})$ with the properties:

(1) $\Gamma_{\sigma}$ starts from $z_{\sigma} \in \Gamma_{0}\left(\theta_{0}\right)$ with $\left|z_{\sigma}\right| \leq 2|\sigma|$ and ends at $\sigma$;

(2) for any $z \in \Gamma_{\sigma},|z| \geq|\sigma|$;

(3) the length of $\Gamma_{\sigma}$ does not exceed $(\lambda+1) \pi^{2}|\sigma|$;

(4) $|w(z)-\mu|>\Delta$ along $\Gamma_{\sigma}$.

Here $T_{0}$ and $\Delta$ are the constants as in Lemma 3.2.

PROOF. The argument in constructing $\Gamma_{\sigma}$ is similar to that of the proof of [11, Lemma 2.3]. In the proof of this lemma, for a curve $\Gamma \ni b, b^{\prime}$, we denote by $\Gamma\left(b, b^{\prime}\right)$ the part of $\Gamma$ from $b$ to $b^{\prime}$. For $\sigma$ as in the lemma, take $s_{0} \in \Gamma_{0}\left(\theta_{0}\right)$ such that $\left|s_{0}\right|=|\sigma|$. Then $\left|w\left(s_{0}\right)-\mu\right| \geq \Delta_{0}>2 \Delta$. We may suppose that $\left|\arg s_{0}-\theta_{0}\right|<\pi / 4$. On $\mathcal{R}(\boldsymbol{C} \backslash\{0\})$, draw the $\operatorname{arc} S: z=|\sigma| e^{i \theta}$ with $\arg s_{0} \leq \theta \leq \arg \sigma\left(\leq \theta_{0}+(\lambda+1) \pi / 2\right)$ or $\left(\theta_{0}-(\lambda+1) \pi / 2 \leq\right)$ $\arg \sigma \leq \theta \leq \arg s_{0}$, which joins $s_{0}$ to $\sigma$. The curve $\Gamma_{\sigma}$ is constructed by step-by-step modification of $S$. If $|w(z)-\mu|>\Delta$ along $S$, then $S$ is the desired curve with the starting point $z_{\sigma}=s_{0}$. Now suppose that $|w(z)-\mu|>\Delta$ is not always valid along $S$. Start from $s_{0}$ and proceed along $S$. Let $s_{1} \in S$ be the first point satisfying $\left|w\left(s_{1}\right)-\mu\right|=\Delta$ that we meet. By virtue of Lemma 3.2, we may draw a circle $c_{1}:\left|z-s_{1}\right|=\delta_{s_{1}}$ such that $|w(z)-\mu| \geq 2 \Delta$ on $c_{1}$ and that $\sigma$ is outside $c_{1}$. Let $\bar{c}_{1}:=\left\{z \in c_{1} ;|z| \geq|\sigma|\right\}$. Consider the case where $\bar{c}_{1} \cap \Gamma_{0}\left(\theta_{0}\right)=\emptyset$. Then $S \cap \bar{c}_{1}$ is written in the form $\left\{b_{1}^{-}, b_{1}^{+}\right\}$, where $b_{1}^{+} \in S\left(s_{1}, \sigma\right)$. Take the $\operatorname{arcs} S\left(b_{1}^{-}, b_{1}^{+}\right) \subset S$ and $\bar{c}_{1}\left(b_{1}^{-}, b_{1}^{+}\right)=\bar{c}_{1}$, and define $S_{1}:=\left(S \backslash S\left(b_{1}^{-}, b_{1}^{+}\right)\right) \cup \bar{c}_{1}\left(b_{1}^{-}, b_{1}^{+}\right)$, which starts from $z_{\sigma}=s_{0}$ and ends at $\sigma$. If $\bar{c}_{1} \cap \Gamma_{0}\left(\theta_{0}\right) \ni b_{0}$, then we take $S\left(s_{0}, b_{1}^{+}\right) \subset S$ with $b_{1}^{+} \in S\left(s_{1}, \sigma\right) \cap \bar{c}_{1}$ and $\bar{c}_{1}\left(b_{0}, b_{1}^{+}\right) \subset \bar{c}_{1}$, and set $S_{1}:=\left(S \backslash S\left(s_{0}, b_{1}^{+}\right)\right) \cup \bar{c}_{1}\left(b_{0}, b_{1}^{+}\right)$, whose starting point $z_{\sigma}=b_{0}$ satisfies $\left|z_{\sigma}\right| \leq|\sigma|+\left|\delta_{s_{1}}\right| \leq 2|\sigma|$. If $|w(z)-\mu|>\Delta$ along $S_{1}$, then $S_{1}$ is the desired curve. Otherwise, restart from $b_{1}^{+}$and proceed along $S_{1}$ until we find the point $s_{2}$ such that $\left|w\left(s_{2}\right)-\mu\right|=\Delta$. Then, by Lemma 3.2 again, we draw a circle $c_{2}:\left|z-s_{2}\right|=\delta_{s_{2}}$ such that $|w(z)-\mu| \geq 2 \Delta$ on $c_{2}$ and that $\sigma$ is outside $c_{2}$. Let $\bar{c}_{2}:=\left\{z \in c_{2} ;|z| \geq|\sigma|\right\}$. If $\bar{c}_{2} \cap \Gamma_{0}\left(\theta_{0}\right)=\emptyset$, then we write $S_{1} \cap \bar{c}_{2}=\left\{b_{2}^{-}, b_{2}^{+}\right\}$with $b_{2}^{+} \in S_{1}\left(s_{2}, \sigma\right)$, and get $S_{2}:=\left(S_{1} \backslash S_{1}\left(b_{2}^{-}, b_{2}^{+}\right)\right) \cup \bar{c}_{2}\left(b_{2}^{-}, b_{2}^{+}\right)$. In the complementary case $\bar{c}_{2} \cap \Gamma_{0}\left(\theta_{0}\right) \ni b_{0}^{\prime}$ as well, a new curve $S_{2}$ may be obtained by the same way as in the second case of $S_{1}$. If $S_{2}$ is not a satisfactory curve, restart from $b_{2}^{+}$, and repeat this procedure. As far as there exists a point $s_{k} \in S_{k-1}$ such that $\left|w\left(s_{k}\right)-\mu\right|=\Delta$, we replace $S_{k-1}$ by a new curve $S_{k}$ obtained by using Lemma 3.2. As a matter of fact we need not repeat this replacement infinitely many times. Indeed, if there exists an infinite sequence of circles $c_{k}:\left|z-s_{k}\right|=\delta_{s_{k}}$ with the properties $\left|w\left(s_{k}\right)-\mu\right|=\Delta$ and $\sum_{k=1}^{\infty} \delta_{s_{k}}<(\lambda+1) \pi$, then, by (3.8), we may choose a sequence $s_{k_{j}} \rightarrow s_{*} \in S$ satisfying $w\left(s_{k_{j}}\right) \rightarrow w_{*} \neq \infty$ and $w^{\prime}\left(s_{k_{j}}\right) \rightarrow \infty$ as $j \rightarrow \infty$, which gives a contradiction $w\left(s_{*}\right)=w_{*}, w^{\prime}\left(s_{*}\right)=\infty$. Therefore, after repeating this procedure finitely many times, we get $\Gamma_{\sigma}$ as in the lemma. 
3.3. Proof of Theorem 2.1 for $a=1$. The assertion of Theorem 2.1 for $a=1$ is immediately derived from the following:

PROpOSITION 3.6. Let $w(z)$ be a solution of $(\mathrm{V})$ (respectively, $\left.\left(\mathrm{V}_{0}\right)\right)$ with $\boldsymbol{A}\left(\mu, \theta_{0}\right)$ as in Theorem 2.1. Then, for every $\sigma \in \mathcal{R}(\boldsymbol{C} \backslash\{0\})$ satisfying $w(\sigma)=1,|\sigma| \geq T_{0}$ and $\left|\arg \left(\sigma-e^{i \theta_{0}} / 2\right)-\theta_{0}\right| \leq \lambda \pi / 2$, there exists a disk $B(\sigma):|z-\sigma|<\varepsilon_{0}$ (respectively, $|z-\sigma|<\varepsilon_{0}|\sigma|^{1 / 2}$ ) such that $w(z) \neq 1$ for $z \in B(\sigma) \backslash\{\sigma\}$, where $\varepsilon_{0}$ is a constant independent of $\sigma$.

The remaining part of this section is devoted to the proof of this proposition. Suppose that $w(z)$ is a solution of $(\mathrm{V})$. Let $\sigma$ be a point as in Proposition 3.6, and let $\Gamma_{\sigma}$ be the curve given by Lemma 3.5. For $t \in \Gamma_{\sigma}$, function (3.11) with $\Gamma\left(z_{0}, t\right)=\Gamma_{\sigma}(t)$ and $z_{0}=z_{\sigma}$ satisfies $E\left(z_{\sigma}, t\right)^{ \pm 1} \ll 1$, because, by Lemma 3.5 ,

$\left|\int_{\Gamma_{\sigma}(t)} \frac{(w(\tau)-1)(w(\tau)+\mu)}{(w(\tau)-\mu)^{2}} \frac{d \tau}{\tau}\right| \ll\left|\int_{\Gamma_{\sigma}(t)} \frac{d \tau}{\tau}\right| \ll \frac{1}{|\sigma|} \int_{\Gamma_{\sigma}(t)}|d \tau| \ll \frac{(\lambda+1) \pi^{2}|\sigma|}{|\sigma|} \ll 1$,

where $\Gamma_{\sigma}(t)$ denotes the part of $\Gamma_{\sigma}$ from $z_{\sigma}$ to $t$. Then (3.10) combined with Lemma 3.4 gives $|\Phi(\mu, \sigma)| \leq K_{0}^{\prime}|\sigma|^{2}$, where $K_{0}^{\prime}>0$ is independent of $\sigma$. Furthermore, using (3.10) and (3.11) with $\Gamma\left(z_{0}, z\right)=[\sigma, z]$, we may show that

$$
|\Phi(\mu, z)| \leq K_{1}|\sigma|^{2}
$$

is valid if $|z-\sigma|<1$ and if $|w(z)-1| \leq \min \{1,|1-\mu| / 2\}$, where $K_{1}>0$ is independent of $\sigma$. We substitute $w(z)=1+v(z)$ into (3.9) to find

$$
v^{\prime}(z)^{2}+f_{1}(z, v(z)) v^{\prime}(z)+1+f_{2}(z, v(z), \Phi(\mu, z))=0,
$$

where

$$
f_{1}(z, v) \ll|z|^{-1}|v|, \quad f_{2}(z, v, \Phi) \ll|v|+|z|^{-2}|v|^{2}|\Phi|
$$

for $|z| \geq 1$ and for $|v| \leq \min \{1,|1-\mu| / 2\}$. Hence, by (3.14),

$$
v^{\prime}(z)= \pm 1+F(z, v(z), \Phi(\mu, z))
$$

with

$$
|F(z, v(z), \Phi(\mu, z))| \leq C_{1}\left(|v(z)|+|\sigma|^{-2}|v(z)|^{2}|\Phi(\mu, z)|\right) \leq C_{1}\left(1+K_{1}\right)|v(z)|
$$

if $|z-\sigma|<1$ and if $|v(z)| \leq \min \{1,|1-\mu| / 2\}$, where $C_{1}>0$ is independent of $\sigma$. Choose $\varepsilon_{1}>0$ so small that $C_{1}\left(1+K_{1}\right) \varepsilon_{1} \leq 1 / 2$ and that $\varepsilon_{1}<(1 / 2) \min \{1,|1-\mu| / 2\}$. Let $\rho_{*}:=\sup \left\{\rho<1 ;|v(z)|<\varepsilon_{1}\right.$ for $\left.|z-\sigma|<\rho\right\}$. Then, from (3.15) it follows that $1 / 2 \leq\left|v^{\prime}(z)\right| \leq 3 / 2$ for $|z-\sigma|<\rho_{*}$, and that $(1 / 2)|z-\sigma| \leq|v(z)| \leq(3 / 2)|z-\sigma|<$ $(3 / 2) \rho_{*}$. If $(3 / 2) \rho_{*}<\varepsilon_{1}$, this contradicts the definition of $\rho_{*}$. Consequently we conclude that $\rho_{*} \geq \varepsilon_{0}:=(2 / 3) \varepsilon_{1}$, and that $(1 / 2)|z-\sigma| \leq|w(z)-1| \leq(3 / 2)|z-\sigma|$ for $|z-\sigma| \leq \varepsilon_{0}$. Thus we obtain $B(\sigma)$ as desired.

In the case where $w(z)$ is a solution of $\left(\mathrm{V}_{0}\right)$, by an analogous argument, we have, instead of (3.14), $|\Phi(\mu, z)| \leq K_{1}|\sigma|$ for $|z-\sigma|<|\sigma|^{1 / 2}$ as far as $|w(z)-1| \leq \min \{1,|1-\mu| / 2\}$. 
Putting $w(z)=1+u(z)^{2}$, we derive, from (3.9), that

$$
u^{\prime}(z)= \pm z^{-1 / 2} / 4+G(z, u(z), \Phi(\mu, z))
$$

with $G(z, u, \Phi) \ll|z|^{-1 / 2}|u|^{2}+|z|^{-1}|u|+|z|^{-3 / 2}|u|^{2}|\Phi|$ for $|z| \geq 1$ and for $|u|^{2} \leq$ $\min \{1,|1-\mu| / 2\}$. Then $U(z):=\sigma^{1 / 2} u(z)$ satisfies

$$
U^{\prime}(z)= \pm 1 / 4+H(z, U(z), \Phi(\mu, z))
$$

with

$$
\begin{aligned}
|H(z, U(z), \Phi(\mu, z))| & \leq C_{2}\left(|\sigma|^{-1}+|\sigma|^{-1 / 2}|U(z)|+|\sigma|^{-2}|U(z)|^{2}|\Phi(\mu, z)|\right) \\
& \leq C_{2}\left(|\sigma|^{-1}+\left(1+K_{1}\right)|\sigma|^{-1 / 2}|U(z)|\right)
\end{aligned}
$$

if $|z-\sigma|<|\sigma|^{1 / 2}$ and if $\left|\sigma^{-1} U(z)^{2}\right| \leq \min \{1,|1-\mu| / 2\}$, where $C_{2}$ is independent of $\sigma$. Choose $\varepsilon_{2}>0$ (and, if necessary, $\left.T_{0}\right)$ so that $C_{2}\left(T_{0}^{-1}+\left(1+K_{1}\right) \varepsilon_{2}\right) \leq 1 / 8$ and that $\varepsilon_{2}^{2}<(1 / 2) \min \{1,|1-\mu| / 2\}$. Then $1 / 8 \leq\left|U^{\prime}(z)\right| \leq 3 / 8$ as far as $|z-\sigma|<\rho_{* *},|\sigma| \geq T_{0}$, where $\rho_{* *}:=\sup \left\{\rho<|\sigma|^{1 / 2} ;\left|\sigma^{-1 / 2} U(z)\right|<\varepsilon_{2}\right.$ for $\left.|z-\sigma|<\rho\right\}$. Using this fact, we find $(1 / 8)^{2}|\sigma|^{-1}|z-\sigma|^{2} \leq|w(z)-1| \leq(3 / 8)^{2}|\sigma|^{-1}|z-\sigma|^{2}$ for $|z-\sigma|<\varepsilon_{0}|\sigma|^{1 / 2}$ with $\varepsilon_{0}:=(8 / 3) \varepsilon_{2}$. Thus we obtain Proposition 3.6.

4. Proofs of Theorem 2.1 for $a \neq 1$ and Theorem 2.2. Let $\lambda$ and $\theta_{0}$ be as in Section 2 , and let $w(z)$ be a solution of $(\mathrm{V})$ or $\left(\mathrm{V}_{0}\right)$. We need the following facts concerning value distribution in a sector (cf. [12, Lemma 4.2 and Proposition 5.1], [13, Lemmas 6.1 and 6.3]).

LEMMA 4.1. For every $a \in C, T_{\lambda}^{\theta_{0}}(r, 1 /(w-a))=T_{\lambda}^{\theta_{0}}(r, w)+O(1)$.

LEMMA 4.2. $m_{\lambda}^{\theta_{0}}(r, 1 /(w-1)) \ll \log r$.

4.1. Proof of Theorem 2.2. From Lemmas 4.1 and 4.2 it follows that

$$
\begin{aligned}
T_{\lambda}^{\theta_{0}}(r, w) & =T_{\lambda}^{\theta_{0}}(r, 1 /(w-1))+O(1) \\
& =m_{\lambda}^{\theta_{0}}(r, 1 /(w-1))+N_{\lambda}^{\theta_{0}}(r, 1 /(w-1))+O(1) \\
& =N_{\lambda}^{\theta_{0}}(r, 1 /(w-1))+O(\log r) .
\end{aligned}
$$

Suppose that $w(z)$ is a solution of (V) with $\boldsymbol{A}\left(\mu, \theta_{0}\right)$ as in Theorem 2.1. Since $\Omega\left(\theta_{0}, \lambda, r\right) \subset$ $\Sigma\left(\theta_{0}, \lambda, r\right)($ cf. (2.1)),

$$
n_{\lambda}^{\theta_{0}}(r, 1 /(w-1)) \leq v_{\lambda}^{\theta_{0}}(r, \infty, 1 /(w-1))=v_{\lambda}^{\theta_{0}}(r, 1, w) \ll r^{2},
$$

where the last evaluation $\ll r^{2}$ is what has been proved in Section 3. This inequality gives $N_{\lambda}^{\theta_{0}}(r, 1 /(w-1)) \ll r^{2-1 / \lambda}+\log r$. Hence, by Lemma 4.1 and (4.1), for each $a \in C \cup\{\infty\}$ we have $N_{\lambda}^{\theta_{0}}(r, a, w) \leq T_{\lambda}^{\theta_{0}}(r, w)+O(1) \ll r^{2-1 / \lambda}+\log r$, which yields the estimates as in Theorem 2.2. A solution of $\left(\mathrm{V}_{0}\right)$ may be treated in a similar way.

4.2. Proof of Theorem 2.1 for $a \neq 1$. Let us return to the proof of Theorem 2.1. We may suppose that $\lambda>1$, since the result for $\lambda \leq 1$ immediately follows from that for $\lambda>1$. Let $a \in C \cup\{\infty\} \backslash\{1\}$. If $w(z)$ satisfies (V), then Theorem 2.2 together with Lemma 4.1 
implies $N_{\lambda}^{\theta_{0}}(r, 1 /(w-a)) \leq T_{\lambda}^{\theta_{0}}(r, 1 /(w-a))=T_{\lambda}^{\theta_{0}}(r, w)+O(1) \ll r^{2-1 / \lambda}$. Combining this with

$$
\begin{aligned}
N_{\lambda}^{\theta_{0}}(2 r, 1 /(w-a)) & \geq \frac{1}{\lambda} \int_{r}^{2 r} \frac{n_{\lambda}^{\theta_{0}}(t, 1 /(w-a))}{t^{1+1 / \lambda}} d t \\
& \geq \frac{n_{\lambda}^{\theta_{0}}(r, 1 /(w-a))}{\lambda} \int_{r}^{2 r} \frac{d t}{t^{1+1 / \lambda}}=r^{-1 / \lambda}\left(1-2^{-1 / \lambda}\right) n_{\lambda}^{\theta_{0}}(r, 1 /(w-a)),
\end{aligned}
$$

we have $n_{\lambda}^{\theta_{0}}(r, 1 /(w-a)) \ll r^{2}$ for each $\lambda>1$. Note that $\Sigma\left(\theta_{0}, \tilde{\lambda} / 2,2^{-\tilde{\lambda} / 2} r\right) \subset \Omega\left(\theta_{0}, \tilde{\lambda}, r\right)$ for $\tilde{\lambda}>2$, which follows from (2.1) with $\lambda=\tilde{\lambda}, \lambda^{\prime}=\tilde{\lambda} / 2$. Then $v_{\tilde{\lambda} / 2}^{\theta_{0}}\left(2^{-\tilde{\lambda} / 2} r, a, w\right) \leq$ $n_{\tilde{\lambda}}^{\theta_{0}}(r, 1 /(w-a))$. We replace $(\tilde{\lambda}, r)$ by $\left(2 \lambda, 2^{\lambda} r\right)$ to obtain $\nu_{\lambda}^{\theta_{0}}(r, a, w) \ll r^{2}$ for $\lambda>1$. For a solution of $\left(\mathrm{V}_{0}\right)$, by using $T_{\lambda}^{\theta_{0}}(r, w) \ll r^{1-1 / \lambda}$, the estimate $\nu_{\lambda}^{\theta_{0}}(r, a, w) \ll r$ is derived in a similar way. Thus Theorem 2.1 is verified.

5. The third Painlevé transcendents. The argument developed above is also applicable to the third Painlevé equation

$$
w^{\prime \prime}=\frac{\left(w^{\prime}\right)^{2}}{w}-\frac{w^{\prime}}{z}+\frac{1}{z}\left(\alpha w^{2}+\tilde{\alpha}\right)+\beta w^{3}+\frac{\tilde{\beta}}{w}
$$

with $\alpha, \tilde{\alpha}, \beta, \tilde{\beta} \in \boldsymbol{C}$. We may suppose both $(\alpha, \beta) \neq(0,0)$ and $(\tilde{\alpha}, \tilde{\beta}) \neq(0,0)$; otherwise this is solvable by quadrature. For $\hat{\mu} \in C \backslash\{0\}$ we say that a solution $w(z)$ satisfies the condition $\boldsymbol{A}_{0}\left(\hat{\mu}, \theta_{0}\right)$ (respectively, $\left.\boldsymbol{A}_{\infty}\left(\hat{\mu}, \theta_{0}\right)\right)$ if there exists a curve $\Gamma_{0}\left(\theta_{0}\right): z=r e^{i \theta(r)}$, $r \geq 1$ such that $\theta(r) \rightarrow \theta_{0}$ as $r \rightarrow+\infty$ and that $w(z)$ and $1 /(w(z)-\hat{\mu})$ (respectively, $1 / w(z)$ and $1 /(w(z)-\hat{\mu}))$ are bounded along $\Gamma_{0}\left(\theta_{0}\right)$. For a solution of (III) as well, we define $\nu_{\lambda}^{\theta_{0}}(r, a, w)$ as in Section 2 .

TheOREM 5.1. Suppose $(\beta, \tilde{\beta}) \neq(0,0)$ (respectively, $\beta=\tilde{\beta}=0$ and $\alpha \tilde{\alpha} \neq 0)$. Let $w(z)$ be a solution of (III) satisfying $\boldsymbol{A}_{0}\left(\mu, \theta_{0}\right)$ or $\boldsymbol{A}_{\infty}\left(\mu, \theta_{0}\right)$ for some $\left(\mu, \theta_{0}\right) \in(\boldsymbol{C} \backslash\{0\}) \times \boldsymbol{R}$ with $\beta \mu^{3}+\tilde{\beta} / \mu \neq 0$ (respectively, $\alpha \mu^{2}+\tilde{\alpha} \neq 0$ ). Then, for each $a \in \boldsymbol{C} \cup\{\infty\}$ and for each $\lambda>0$,

$$
\begin{array}{cl}
v_{\lambda}^{\theta_{0}}(r, a, w) \ll r^{2} & \text { (respectively, } \left.v_{\lambda}^{\theta_{0}}(r, a, w) \ll r\right), \\
T_{\lambda}^{\theta_{0}}(r, w) \ll r^{2-1 / \lambda}+\log r & \text { (respectively, } \left.T_{\lambda}^{\theta_{0}}(r, w) \ll r^{1-1 / \lambda}+\log r\right)
\end{array}
$$

as $r \rightarrow+\infty$, where the implied constants depend on $\lambda$, and $\sigma_{\lambda}^{\theta_{0}}(a, w) \leq \max \{2-1 / \lambda, 0\}$ (respectively, $\left.\sigma_{\lambda}^{\theta_{0}}(a, w) \leq \max \{1-1 / \lambda, 0\}\right)$.

5.1. Outline of the proof of Theorem 5.1. We may suppose that $w(z)$ satisfies $\boldsymbol{A}_{0}\left(\mu, \theta_{0}\right)$ along $\Gamma_{0}\left(\theta_{0}\right)$, because $1 / w(z)$ is a solution of (III) with $(-\tilde{\alpha},-\alpha,-\tilde{\beta},-\beta)$. Using $\beta \mu^{3}+\tilde{\beta} / \mu \neq 0$ or $\alpha \mu^{2}+\tilde{\alpha} \neq 0$, we have the following instead of Lemma 3.2.

Lemma 5.2. Suppose $(\beta, \tilde{\beta}) \neq(0,0)$ (respectively, $\beta=\tilde{\beta}=0$ and $\alpha \tilde{\alpha} \neq 0$ ), and set $\Delta_{0}:=\inf \left\{|w(z)-\mu| ; z \in \Gamma_{0}\left(\theta_{0}\right)\right\}$. Then there exist positive numbers $T_{0}$ and $\Delta \leq \Delta_{0} / 3$ such that the following assertions are valid for every $s \in \mathcal{R}(\boldsymbol{C} \backslash\{0\})$ satisfying $|s| \geq T_{0}$ : 
(1) if $|w(s)-\mu| \leq \Delta$, then $|w(z)-\mu| \geq 2 \Delta$ on the circle $|z-s|=\delta_{s}$;

(2) $w(z) \neq 0, \infty$ in the disk $|z-s| \leq \delta_{s}$.

Here $\delta_{s}$ is some positive number satisfying $\delta_{s}^{-1} \leq C_{0}\left(1+\left|w^{\prime}(s)\right|\right), \delta_{s} \leq C_{0}$ (respectively, $\left.\delta_{s}^{-1} \leq C_{0}|s|^{-1 / 2}\left(1+|s|^{-1 / 2}\left|w^{\prime}(s)\right|\right), \delta_{s} \leq C_{0}|s|^{1 / 2} \leq|s|\right), C_{0}>0$ being independent of $s$.

Instead of (3.9) we employ

$$
\Phi(\mu, z):=\Psi(z)+\frac{4 z w^{\prime}(z)}{w(z)-\mu}
$$

with

$$
\Psi(z):=\frac{z^{2} w^{\prime}(z)^{2}}{w(z)^{2}}-\frac{2 z w^{\prime}(z)}{w(z)}-2 \alpha z w(z)-\beta z^{2} w(z)^{2}+\frac{2 \tilde{\alpha} z}{w(z)}+\frac{\tilde{\beta} z^{2}}{w(z)^{2}}
$$

(cf. [7], [10]). These functions satisfy

$$
\begin{aligned}
& \Phi^{\prime}(\mu, z)+\frac{4 \mu z^{-1} w(z)}{(w(z)-\mu)^{2}} \Phi(\mu, z)=-8 \mu\left(\frac{w(z)}{(w(z)-\mu)^{2}}\right)^{\prime} \\
&-\frac{4}{(w(z)-\mu)^{2}}\left((\alpha \mu w(z)-\tilde{\alpha})(w(z)+\mu)+\beta \mu^{2} z w(z)^{2}-\tilde{\beta} z\right), \\
& \Psi^{\prime}(z)=-4 \alpha w(z)-4 \beta z w(z)^{2},
\end{aligned}
$$

which yield integral expressions for $\Phi(\mu, z)$ and $\Psi(z)$ corresponding to (3.10) and (3.13), respectively. Using them together with $\boldsymbol{A}_{0}\left(\mu, \theta_{0}\right)$, we may prove the following facts instead of Lemmas 3.4 and 3.5:

Lemma 5.3. Suppose $(\beta, \tilde{\beta}) \neq(0,0)$ (respectively, $\beta=\tilde{\beta}=0$ and $\alpha \tilde{\alpha} \neq 0)$. Then there exists a positive number $K_{0}$ such that $|\Phi(\mu, z)| \leq K_{0}|z|^{2}$ (respectively, $|\Phi(\mu, z)| \leq$ $\left.K_{0}|z|\right)$ along $\Gamma_{0}\left(\theta_{0}\right)$.

LEMMA 5.4. For every pole or zero $\sigma \in \mathcal{R}(\boldsymbol{C} \backslash\{0\})$ satisfying $|\sigma| \geq T_{0}$ and $\mid \arg (\sigma-$ $\left.e^{i \theta_{0}} / 2\right)-\theta_{0} \mid \leq \lambda \pi / 2$, there exists a curve $\Gamma_{\sigma} \subset \mathcal{R}(\boldsymbol{C} \backslash\{0\})$ with the same properties as in Lemma 3.5, where $T_{0}$ and $\Delta$ are as in Lemma 5.2.

By these lemmas we have

Proposition 5.5. (1) Suppose $\beta \neq 0$ (respectively, $\beta=\tilde{\beta}=0$ and $\alpha \tilde{\alpha} \neq 0$ ). Then, for every pole $\sigma \in \mathcal{R}(\boldsymbol{C} \backslash\{0\})$ satisfying $|\sigma| \geq T_{0}$ and $\left|\arg \left(\sigma-e^{i \theta_{0}} / 2\right)-\theta_{0}\right| \leq \lambda \pi / 2$, there exists a disk $B(\sigma):|z-\sigma|<\varepsilon_{0}$ (respectively, $|z-\sigma|<\varepsilon_{0}|\sigma|^{1 / 2}$ ) such that $w(z) \neq \infty$ for $z \in B(\sigma) \backslash\{\sigma\}$, where $\varepsilon_{0}$ is a constant independent of $\sigma$.

(2) Under the supposition $\tilde{\beta} \neq 0$, for every zero $\sigma \in \mathcal{R}(\boldsymbol{C} \backslash\{0\})$ satisfying $|\sigma| \geq T_{0}$ and $\left|\arg \left(\sigma-e^{i \theta_{0}} / 2\right)-\theta_{0}\right| \leq \lambda \pi / 2$, there exists a disk $B(\sigma):|z-\sigma|<\varepsilon_{0}$ such that $w(z) \neq 0$ for $z \in B(\sigma) \backslash\{\sigma\}$.

This is derived by an argument analogous to that in the proof of Proposition 3.6. For instance, in the case where $\beta=\tilde{\beta}=0$ and $\alpha \tilde{\alpha} \neq 0$, we have

$$
w^{\prime}(z)= \pm(2 \alpha)^{1 / 2} z^{-1 / 2} w(z)^{3 / 2}+O\left(\left|z^{-1 / 2} w(z)\right|+\left|z^{-3 / 2} w(z)^{1 / 2} \Phi(\mu, z)\right|\right)
$$


if $|w(z)|$ and $|z|$ are sufficiently large. Then, by virtue of Lemmas 5.3 and 5.4, for every pole $\sigma$ such that $|\sigma|$ is sufficiently large, the function $U(z)=\sigma^{1 / 2} w(z)^{-1 / 2}$ satisfies $1 / 2 \leq$ $|\alpha / 2|^{-1 / 2}\left|U^{\prime}(z)\right| \leq 3 / 2$ if $|z-\sigma|<|\sigma|^{1 / 2}$ and if $\left|\sigma^{-1 / 2} U(z)\right|<\varepsilon_{3}$, where $\varepsilon_{3}$ is independent of $\sigma$. Using this fact, we obtain the disk $B(\sigma):|z-\sigma|<\varepsilon_{0}|\sigma|^{1 / 2}$.

As an immediate consequence of this proposition, if, for example, $(\beta, \tilde{\beta}) \neq(0,0)$, we have $v_{\lambda}^{\theta_{0}}(r, \infty, w) \ll r^{2}$ or $v_{\lambda}^{\theta_{0}}(r, 0, w) \ll r^{2}$, and hence $N_{\lambda}^{\theta_{0}}(r, w) \ll r^{2-1 / \lambda}+\log r$ or $N_{\lambda}^{\theta_{0}}(r, 1 / w) \ll r^{2-1 / \lambda}+\log r$. This yields $T_{\lambda}^{\theta_{0}}(r, w) \ll r^{2-1 / \lambda}+\log r$ (cf. [12, p. 103 and Section 5.2]), which is also verified by using $m_{\lambda}^{\theta_{0}}(r, w) \ll \log r$ or $m_{\lambda}^{\theta_{0}}(r, 1 / w) \ll \log r$. Then we obtain Theorem 5.1 by the same argument as in Section 4.2.

\section{REFERENCES}

[ 1 ] F. V. ANDREEV AND A. V. KitAEV, Exponentially small corrections to divergent asymptotic expansions of solutions of the fifth Painlevé equation, Math. Res. Lett. 4 (1997), 741-759.

[ 2 ] F. V. Andreev And A. V. KitAev, Connection formulae for asymptotics of the fifth Painlevé transcendent on the real axis, Nonlinearity 13 (2000), 1801-1840.

[ 3 ] G. BARSEGIAN, I. LAINE AND D. T. LÊ, On topological behaviour of solutions of some algebraic differential equations, Complex Var. Elliptic Equ. 53 (2008), 411-421.

[4] A. A. Goldberg And I. V. OstrovskiI, Value distribution of meromorphic functions, Translations of Mathematical Monographs 236, American Mathematical Society, Providence, 2008.

[ 5 ] B. JA. LEVIN AND I. V. OstrovskiI, On the dependence of the growth of an entire function on the distribution of the zeros of its derivatives (in Russian), Sibirsk. Mat. Zh. 1 (1960), 427-455; English translation in: Amer. Math. Soc. Transl. 32 (1963), 323-357.

[6] Y. SASAKI, Value distribution of the fifth Painlevé transcendents in sectorial domains, J. Math. Anal. Appl. 330 (2007), 817-828.

[ 7 ] Y. SASAKI, Value distribution of the third Painlevé transcendents in sectorial domains, Proc. Japan Acad. Ser. A Math. Sci. 83 (2007), 79-82.

[ 8 ] S. Shimomura, Analytic integration of some nonlinear ordinary differential equations and the fifth Painlevé equation in the neighbourhood of an irregular singular point, Funkcial. Ekvac. 26 (1983), 301-338.

[9] S. Shimomura, On solutions of the fifth Painlevé equation on the positive real axis. II, Funkcial. Ekvac. 30 (1987), 203-224.

[10] S. Shimomura, Proofs of the Painlevé property for all Painlevé equations, Japan. J. Math. 29 (2003), 159180.

[11] S. Shimomura, Growth of modified Painlevé transcendents of the fifth and the third kind, Forum Math. 16 (2004), 231-247.

[12] S. Shimomura, Equi-distribution of values for the third and the fifth Painlevé transcendents, Nagoya Math. J. 192 (2008), 89-109.

[13] S. Shimomura, Truncated solutions of the fifth Painlevé equation, Funkcial. Ekvac. 54 (2011), 451-471.

[14] K. TAKano, A 2-parameter family of solutions of Painlevé equation (V) near the point at infinity, Funkcial. Ekvac. 26 (1983), 79-113.

[15] M. TsuJi, On Borel's directions of meromorphic functions of finite order, Tohoku Math. J. (2) (1950), 97-112.

[16] S. WANG, On the sectorial oscillation theory of $f^{\prime \prime}+A(z) f=0$, Ann. Acad. Sci. Fenn. Ser. A I Math. Dissertationes 92 (1994).

[17] S. YoshidA, 2-parameter family of solutions for Painlevé equations (I)-(V) at an irregular singular point, Funkcial. Ekvac. 28 (1985), 233-248. 
DEPARTMENT OF MATHEMATICS

KEIO UNIVERSITY

3-14-1, HiYOSHI

KOHOKU-KU YOKOHAMA 223-8522

JAPAN

E-mail address: shimomur@math.keio.ac.jp 